\title{
A sparse particle filter for indoor localization using mobile phones
}

\author{
Congwei Dang ${ }^{\text {a) }}$ and Kaoru Sezaki \\ Institute of Industrial Science, The University of Tokyo \\ 4-6-1 Komaba, Meguro-ku, Tokyo 153-8505, Japan \\ a)dangcongwei@mcl.iis.u-tokyo.ac.jp
}

\begin{abstract}
In this paper, we propose a novel sparse particle filter applied for indoor pedestrian localization. Unlike the traditional particle filters that usually contain thousands of particles, this algorithm uses one or several particle sequences in location tracking. The particle is split to cover all the possible tracks when matching ambiguity occurs. This algorithm can significantly reduce the computation burden as well as energy consumption. Field experiments are conducted using mobile phones and the results show that the proposed method can perform localization with much higher efficiency, high reliability, and merely slight loss of accuracy.
\end{abstract}

Keywords: indoor localization, signal fingerprint, particle filter, sensor fusion

Classification: Navigation, Guidance and Control Systems

\section{References}

[1] Vision Statement, "How People Really Use Mobile," Harvard Business Review, pp. 30-31, Jan.-Feb. 2013.

[2] D. Ahlers, M. Pielot, D. Wichmann, and S. Boll, "GNSS quality in pedestrian applications - a developer perspective," Proceedings of the 5th Workshop on Positioning, Navigation and Communication 2008 (WPNC '08), pp. 45-54, 2008.

[3] D. Simon, Optimal State Estimation, John Wiley \& Sons, Inc., New Jersey, USA, 2006.

[4] C. Dang, K. Sezaki, and M. Iwai, "DECL: A Circular Inference Method for Indoor Pedestrian Localization Using Phone Inertial Sensors," The 7th International Conference on Mobile Computing and Ubiquitous Networking (ICMU 2014), pp. 117-122, 2014.

[5] H. Wang, S. Sen, A. Elgohary, M. Farid, M. Youssef, and R. R. Choudhury, "No Need to War-Drive: Unsupervised Indoor Localization," Proceedings of the 10th International Conference on Mobile Systems, Applications, and Services (MobiSys '12), pp. 197-210, 2012.

[6] Y. Kim, H. Shin, and H. Cha, "Smartphone-based Wi-Fi PedestrianTracking System Tolerating the RSS Variance Problem," 2012 IEEE International Conference on Pervasive Computing and Communications (PerCom 2012), pp. 11-19, 2012. 


\section{Introduction}

Location information plays an important role in modern mobile applications. In a survey [1] it is found that users spend most time with mobile phones in various context dependent activities. This requires the applications should be context aware and therefore user location becomes an indispensable primitive. Although GPS is the de facto standard localization method in most outdoor environments, it can hardly be used in many indoor spaces due to the highly deteriorated signals [2].

Recently, localization using mobile phones has attracted great research attention. Traditional particle filter [3] is widely used to fuse various phone sensors for this purpose. However, a major issue of particle filter is its efficiency. In practice it needs to maintain thousands of particles to obtain the required accuracy and reliability. This is especially problematic for mobile applications, because such intensive computation burden not only delays the response time of user interface but also increases the energy consumption so that shortens the battery life.

In this paper, we propose a novel sparse particle filter that is designed to efficiently reduce the computation burden and simultaneously maintain the accuracy and reliability as much as possible. The sparse particle filter has three salient features: (1) in normal cases only one particle is used to represent the pedestrian location, (2) when tracking ambiguity occurs, the particle can be split to tolerate such situation, and (3) unlike the traditional particle filter that uses one-step estimation, it can apply multi-step optimal estimation to improve the accuracy, since low computation burden saves enough CPU resources for such kind of processing.

\section{Sparse particle filter}

In general, traditional particle filter continuously performs a two-phase estimation process to track the object location, including a a priori phase in which particle locations are estimated and a a posteriori phase in which particle likelihoods are computed and particles are resampled based on the such likelihoods.

In sparse particle filtering presented in this paper, an alternative method is proposed to manipulate the particle. In normal cases only one particle is used to represent the current location. We update the particle using a localization method combining pedestrian dead reckoning (PDR) and real-time learning as showed in our previous work [4]. This method uses graph-based maps that are constituted of nodes and edges. Map-matching techniques are applied to correct the estimated location. Landmarks such as intersections and elevators are used to trigger the learning procedure. In this paper, signal fingerprints such as Wi-Fi and magnetic signatures [5] are also used as the triggers of learning.

To explain the learning processing, assume $y_{k}$ is the ground truth obtained from the newly detected landmark or signal fingerprint at the $k$-th step, $x_{k-M+1}, \cdots, x_{k-1}, x_{k}$ is the sequence of location data of $M$ steps after the 
previous learning, and $P=\left[p_{1}, p_{2}, \cdots, p_{d}\right]^{T}$ is a vector of system parameters relevant to PDR module [4]. Then the optimal value $P^{*}$ can be computed via the following optimization equation:

$$
P^{*}=\underset{P}{\arg \min } f\left(y_{k}, x_{k}, \cdots, x_{k-M+1}, P\right)
$$

In PDR tracking ambiguity often occurs due to the errors. For example, if several intersections are very close to one another and the pedestrian turns through one of them, it might be very difficult to find the correct one due to the estimation errors. Also, the correct forward walking direction might be hard to find due to the measure errors even when the intersection is correctly determined.

In sparse particle filter, we split the original particle into multiple particles to cover such ambiguities. If and only if the place or direction can be matched based on the present estimation, a new particle will be created to cover this match. Then, each particle is updated independently with newly read measurements. After detecting a new landmark or signal fingerprint the particle sequences are checked and non-matched ones will be discarded.

We manage error range information for each particle. Such information is crucial for map-matching. The estimation error is assumed to obey bivariate Gaussian distribution $\mathcal{N}\left(\mu_{1}, \mu_{2}, \sigma_{1}^{2}, \sigma_{2}^{2}, \rho\right)$ in the $x y$-plane as follows:

$$
f(x, y)=\frac{1}{2 \pi \sigma_{1} \sigma_{2} \sqrt{1-\rho^{2}}} e^{-\frac{1}{2\left(1-\rho^{2}\right)}\left[\left(\frac{x-\mu_{1}}{\sigma_{1}}\right)^{2}-2 \rho\left(\frac{x-\mu_{1}}{\sigma_{1}}\right)\left(\frac{y-\mu_{2}}{\sigma_{2}}\right)+\left(\frac{y-\mu_{2}}{\sigma_{2}}\right)^{2}\right]}
$$

We assume $\mu_{1}=\mu_{2}=\rho=0$, and $\sigma_{1}=\sigma_{2}=\sigma_{L}$. Parameter $\sigma_{L}$ is called the error indicator of the particle. During the location updates when no landmark or signal fingerprint is detected, we singly use PDR for localization and consequently the error will continuously increase. We enlarge the value of $\sigma_{L}$ appropriately after each update to represent such drift phenomenon.

In signal fingerprint matching, for a signal fingerprint $S$, we assume the measurement noise also obeys to the bivariate normal distribution showed in Equation (2), and assume $\mu_{1}=\mu_{2}=\rho=0$, and $\sigma_{1}=\sigma_{2}=\sigma_{S}$. Similarly, $\sigma_{S}$ is the error indicator of $S$ that describes its location quality.

Assume the present estimated location of the particle is $L$, and its error indicator is $\sigma_{L}$, after detecting the signal fingerprint $S$ with an error indicator $\sigma_{S}$, we determine the optimal matched location $L^{\prime}$ that has the maximum probability density. Suppose the distance from $L$ to $S$ is $D$, then we can show $L^{\prime}$ is on the line segment from $L$ to $S$ and the distance from $L$ to $L^{\prime}$ is $r^{*}$ :

$$
r^{*}=\frac{\sigma_{L}^{2} D}{\sigma_{L}^{2}+\sigma_{S}^{2}}
$$

After a successful match the value of $\sigma_{L}$ is reset to the initial value since the drift has been cleared.

\section{Evaluations}

We develop a prototype system running on Android ${ }^{\mathrm{TM}}$ mobile phones. We implement the sparse particle filter reusing our previous developed PDR mod- 
ule [4]. In addition, we also implement the traditional particle filter that is used for the comparison. The number of the particles is set to 1000 in the evaluations.

We conduct field experiments in three test-beds including a shopping mall, a subway station, and a research building. Test results from the sparse particle filter are compared to the results produced by the single PDR module and also those from the traditional particle filter.

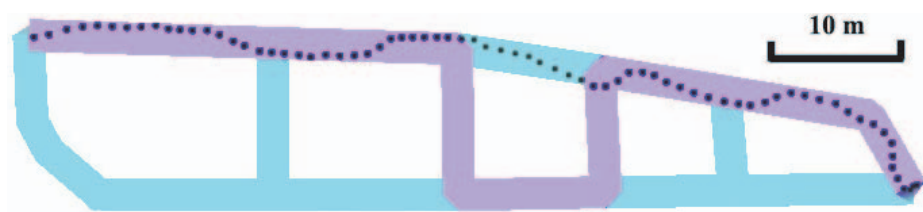

(a) Result from the single PDR module

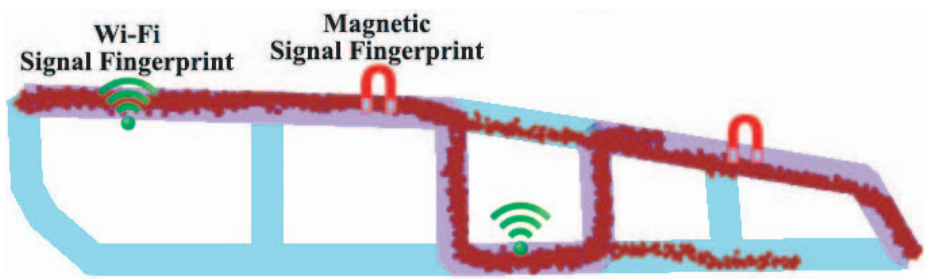

(b) Result from the traditional particle filter

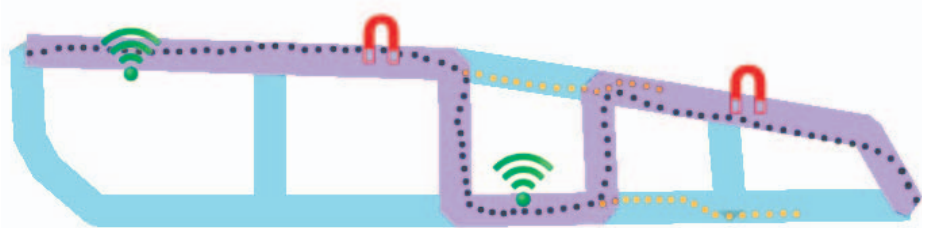

(c) Result from the sparse particle filter

Fig. 1. Localization results produced by the three methods.

Fig. 1 shows the localization results produced by the three methods respectively. They take the same sensor data as the input. The pedestrian holds a phone in hand and walks from left to right. The real path is filled in the dark color. The top diagram shows the tracking points estimated using the single PDR module. The direction at the second intersection is mismatched. The middle diagram shows the result from the traditional particle filter (for the sake of view only a portion of particles are displayed). The particles are split into sub-groups at the intersections when more than one candidate direction exists. The particles that go into wrong directions are discarded and resampled after detecting the signal fingerprints. The bottom diagram shows the result of the sparse particle filter. Since the particle is split in the ambiguous matched places, the result is similar to the traditional particle filter but with much fewer particles.

In some cases a particle cannot detect the signal fingerprint registered in the database. One possible situation is that the particle is in the wrong place, while another highly possible condition is that the signal sources such 
as Wi-Fi access points are turned off. Since it is difficult to distinguish such two situations we do not use these cases to check the validity of the particle.

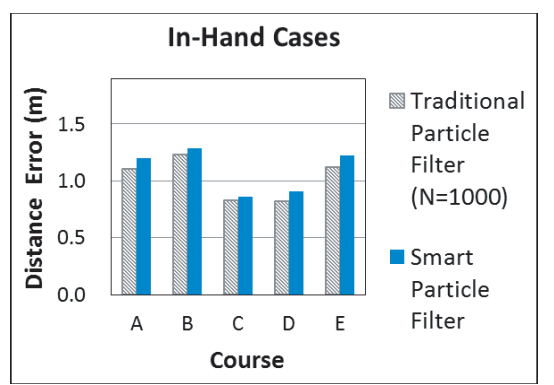

(a) In-hand cases

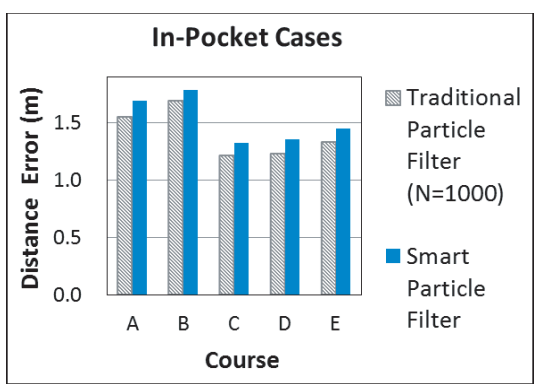

(b) In-pocket cases

Fig. 2. Accuracy performance in terms of distance error.

Then we check the accuracy performance. We select five courses and compare the results of the two filters. The average distance errors of the sparse particle filter merely increase by $7.9 \%$ and $8.3 \%$ compared to the traditional particle filter for in-hand cases and in-pocket cases respectively. The results are showed in Fig. 2.

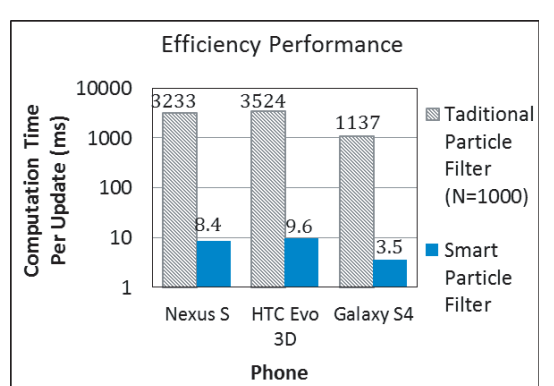

(a) Computation efficiency

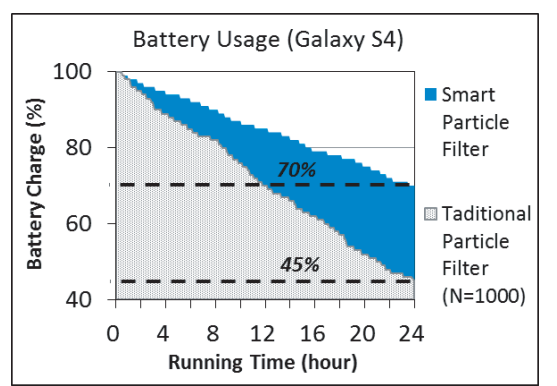

(b) Battery usage

Fig. 3. Computational performance.

Next, we investigate the average computation time of one update of location. Three models of mobile phones are used in the evaluation, including Nexus S, HTC Evo 3D, and Galaxy S4. The results show that the efficiency is increased by about 300 times compared to the traditional particle filter.

We perform simulation experiments to check the phone battery usage. The phones are fully charged in advance. Then the localization program is executed repeatedly taking a set of sensor data of a walking course as the input. The traditional particle filter and sparse particle filter are applied separately and the energy usage logs are checked. For Galaxy S4 after the first 24 hours, the energy consumption rate using sparse particle filter is improved by $45 \%$ compared to using traditional particle filter. Fig. 3 shows the evaluation results of computation efficiency and battery usage. 


\section{Conclusion and Discussion}

In this paper we have presented a novel sparse particle filter that is appropriate for indoor localization applications using mobile phones. The evaluation results show that the proposed method has much higher efficiency, high reliability, and merely loses slight accuracy compared to the traditional method.

It is reported that Wi-Fi fingerprints suffer serious variance problems [6] therefore are unstable. Consequently in many indoor spaces great efforts are required to build and maintain a comprehensive fingerprint database. In literature [5] an unsupervised learning approach is claimed for building the fingerprint database. In such cases the density of fingerprints is usually relatively low therefore singly using fingerprint method can hardly obtain satisfied performance. Our method provides a feasible solution for localization applications in real world that can effectively and efficiently incorporate PDR and fingerprinting technologies. 